\title{
Kingella oralis
}

National Cancer Institute

\section{Source}

National Cancer Institute. Kingella oralis. NCI Thesaurus. Code C86452.

A species of aerobic or facultatively anaerobic, Gram negative, rod and coccobacilli shaped bacteria assigned to the phylum Proteobacteria. This species is nonmotile, oxidase positive and catalase negative, and ferments glucose. $\mathrm{K}$. oralis is normally found in small numbers in the oral cavity; periodontitis and ging ivitis may correlate with increases in numbers of this species in the gingiva. 